\title{
Effect of iron chelation on inflammatory joint disease
}

\author{
F J ANDREWS, ${ }^{1}$ C J MORRIS, ${ }^{1}$ G KONDRATOWICZ, ${ }^{2}$ AND D R BLAKE ${ }^{1}$ \\ From the ${ }^{1}$ Rheumatism Research Wing and the ${ }^{2}$ Department of Pathology, The Medical School, University of \\ Birmingham
}

SUMMARY Mild iron deficiency induced by treatment with the iron chelator desferrioxamine reduced the incidence and severity of joint inflammation associated with adjuvant disease in rats but did not alter the local primary inflammatory response or the systemic sequelae. Both rats treated with desferrioxamine and control rats showed increased levels of $\alpha_{1}$ acid glycoprotein, characteristic pathological changes in the liver and inguinal lymph nodes, and the primary inflammatory response at the site of injection. Histology, microfocal radiography, and gross assessment of the joints, however, showed a suppression or reduction in soft tissue swelling and bone erosion in the rats treated with desferrioxamine. This is a novel observation suggesting an apparently selective influence of iron on joint mediated inflammation.

Key words: inflammation, arthritis.

Any sustained inflammatory reaction causes significant changes in iron metabolism with a drop in serum iron and a redistribution of iron to the activated reticuloendothelial system. Muirden and Senator were one of the first to suggest that in rheumatoid arthritis, iron within the synovium (an extension of the reticuloendothelial system) may exacerbate the inflammatory reaction. ${ }^{1}$ This view has received support from several clinical observations: in early rheumatoid arthritis high levels of synovial iron anticipated a poor prognosis. ${ }^{2}$ Iron dextran treatment will exacerbate rheumatoid disease, and the effect appears to be mediated by iron (rather than the dextran component), involving the promotion of lipid peroxidation. ${ }^{3}$ To investigate further the possible role of iron in joint inflammation we have studied the effect of iron chelation using desferrioxamine (DFX) in adjuvant disease in rats. Desferrioxamine has been used successfully in reducing both acute 4 and chronic ${ }^{5}$ inflammation.

Adjuvant disease was selected as an appropriate model to continue our studies as iron deposits are present within the inflamed synovium, ${ }^{6}$ and the histological features of the adjuvant synovium are a reasonably close approximation to those found in rheumatoid arthritis. Erosive bone damage appears to be a consequence of this inflammation. In

Accepted for publication 26 September 1986.

Correspondence to Dr D R Blake, Rheumatism Research Wing, The Medical School, Birmingham B15 2TJ. addition, adjuvant disease has a 'systemic' extraarticular component which also has some features in common with the extra-articular features of rheumatoid disease. ${ }^{7}$

\section{Materials and methods}

Male inbred Wistar rats $(200 \mathrm{~g})$ were obtained from Bantin and Kingman (Hull, UK). Adjuvant arthritis was induced on day 0 using the method of Kaibara et al. ${ }^{8}$ All rats received a single intradermal injection in the base of the tail of $0.1 \mathrm{ml}$ of a $10 \mathrm{mg} / \mathrm{ml}$ suspension of Mycobacterium butyricum (Difco, Surrey, UK) in liquid paraffin oil. Animals were killed on days 18,22 , or 34 depending upon the extent of joint inflammation.

\section{IRON LEVELS}

Rats were divided into five groups of 12 . Group A received daily intraperitoneal injections of 100 $\mathrm{mg} / \mathrm{kg}$ DFX from day 0 . Group B received the same treatment but from day 12 (found from previous experiments to be the usual time of onset of joint inflammation). Group $\mathrm{C}$ received daily intraperitoneal injections of $200 \mathrm{mg} / \mathrm{kg}$ DFX from day 0 and group $D$ the same treatment from day 12 . The control group $\mathrm{E}$ received daily intraperitoneal injections of sterile saline, the diluent for the DFX.

In all animals the haemoglobin levels were assessed at the time of death by a cyanomethaemoglobin technique (Sigma, USA). Liver iron stores were 
assessed histochemically using Perls' Prussian blue stain based on the method described by Drury and Wallington. ${ }^{9}$ The results of the Perls' technique were controlled using known positive sections. Factors which could affect the intensity of the reaction such as staining time and ferrocyanide concentration were strictly standardised. A subjective score of $0-4$ was allocated to each section of rat liver corresponding to the level of stainable iron. With a score of 0 there was a total absence of stainable iron and a score of 4 corresponded to $>30$ Perls' positive cells per high power field chosen at random.

\section{LOCAL INFLAMMATION}

The development of an inflammatory focus at the site of injection of adjuvant was noted. The extent of reaction was assessed by measuring the diameter of the lesion and by histological assessment of the haematoxylin/eosin $(\mathrm{H} / \mathrm{E})$ stained sections of the tail. As we have noted that the primary inflammatory lesion is associated with periostitis of underlying vertebrae at the base of the tail this was assessed by microfocal radiography (see below).

\section{JOINT INFLAMMATION}

Joint inflammation was assessed by an established method based on the foot scoring system first described by Currey and Ziff. ${ }^{10}$ A subjective score of $0-4$ was allocated to each hind foot depending upon the extent of the inflammation, with a maximum score of 8 per rat. Zero represented no inflammation, 1 represented slight redness and swelling of the foot, 2 represented swelling of the foot such that the tendons were no longer visible, 3 represented swelling extending to the ankle joint, and a score of 4 represented gross inflammation and deformity of the ankle joint.

At the time of death both back legs were removed and fixed in buffered formal saline. Joints were randomly selected for histology and radiography. Where rat ankle joints were selected for histology, radiography was carried out on the opposite ankle joint from the same rat.

\section{Histology}

Ankle joints were decalcified in $10 \%$ formic acid and processed using a double embedding technique. Paraffin wax sections $(5 \mu \mathrm{m})$ were cut and stained with $\mathrm{H} / \mathrm{E}$. Sections were assessed by two observers.

\section{Microfocal radiography}

The $x$ ray system used in this work comprised a demountable $x$ ray unit, an X-Y positioning frame, and Marconi image intensifier and monitor. The special $25 \mu \mathrm{m}$ microfocal $x$ ray set had an effective resolution of $>20 \quad 1 \mathrm{p} / \mathrm{mm}$. Radiographs werह obtained at a magnification of $\times 10$ for the feet and $\times 4$ for the tails using standard $x$ ray cassettes positioned in front of the image intensifier. Expog sures were $60 \mathrm{kV}$ and $1 \mathrm{mAS}(0.5 \mathrm{~mA})$.

Radiographs were assessed by two observers. $\frac{\bar{m}}{\frac{1}{2}}$

SYSTEMIC DISEASE

Lymph nodes

Several studies have shown that lymph nodes have $\vec{Q}$ role in the draining of the site of inoculation in the early phase of development of adjuvant disease. ${ }^{11} \mathrm{~g}$ The inguinal lymph nodes were removed from a: the rats at the time of death and were processed by $\frac{2}{2}$ standard histological procedure. The hyperplastie response of the lymph nodes to adjuvant was assessed semiquantitatively by measuring the maxi mum diameter of the nodes and determining the number of follicles with germinal centre formation. The presence or absence of granuloma formation paracortical hyperplasia, sinus histiocytosis, an dilatation were recorded. Sections of lymph node were stained for Perls' iron as described above.

\section{Liver}

Samples of liver were removed from all the rats ans processed by a standard histological procedure. Tho presence of foci of inflammatory cells or granulö mas, or both, in the liver are a feature of adjuvan disease. $.^{13} \mathrm{H} / \mathrm{E}$ sections of liver from all the rats wer $\overrightarrow{8}$ assessed and the presence/absence of these features recorded in 10 high power fields of the section selected at random.

$\alpha_{1}$ Acid glycoprotein

$\alpha_{1}$ Acid glycoprotein levels were assessed in the plasma from all the rats at the time of death by an immunodiffusion method. ${ }^{14}$ Antiserum, pooled acute and pooled normal rat plasma were obtaine from Dr M Billingham (Eli Lilley, Surrey, UKg Inter- and intraplate variation were calculated using pooled acute and normal rat plasma with know levels of $\alpha_{1}$ acid glycoprotein. Normal levels of $\alpha_{p}$ acid glycoprotein were obtained from the plasma of control rats of the same age, strain, and sex as the test rats.

STA TISTICS

Analysis of parametric data was carried out usin Student's $t$ test and the data are expressed as the mean (SD). Non-parametric data were analysed using a Mann-Whitney $U$ test.

\section{Results}

IRON LEVELS

Iron chelation with DFX did not significantly affeet 
haemoglobin levels (100 mg/kg DFX: 167 (SD $16 \mathrm{~g} / \mathrm{l}$, $200 \mathrm{mg} / \mathrm{kg}$ DFX: 162 (17) g/l, saline: 159 (14) g/l; Student's $t$ test). Control rats with no adjuvant disease but of the same age, strain, and sex had haemoglobin levels of 169 (2) g/l. DFX treatment reduced iron stores. Sixty two per cent of rats treated with DFX (irrespective of dose) had no stainable iron present in the liver, the remaining $38 \%$ had very low levels (grade 1). All the control rats had evidence of stainable iron in the liver: $29 \%$ had grade 1 levels, $42 \%$ grade 2 , and $29 \%$ grade 3 .

\section{LOCAL INFLAMMATION}

All the rats developed a primary inflammatory focus at the site of injection in the base of the tail. There was no significant difference in the diameter of the lesion (DFX $100 \mathrm{mg} / \mathrm{kg}$ : 9.0 (SD 5.9) mm, DFX $200 \mathrm{mg} / \mathrm{kg}$ : $14.2(8.7) \mathrm{mm}$, saline: $10.0(6.2) \mathrm{mm}$; Student's $t$ test).

Histological investigation of $\mathrm{H} / \mathrm{E}$ stained sections showed the lesion consisted of ulceration of the epidermis with underlying granulation tissue formation. The granulation tissue contained a mixed inflammatory cell infiltrate consisting mainly of lymphocytes, histiocytic cells with some polymorphs, and occasional Langhans' type giant cells.

Microfocal radiographs of the tail showed an intense periostitis, florid new bone formation, and evidence of bone destruction in the vertebrae underlying the injection site. Both DFX treated and control rats had the same type and extent of reaction at the injection site as assessed histologically and by microfocal radiography.

\section{JOINT IN FLAMMATION}

Initial signs of arthritis were noted from day 12 . Between days 12 and 13 there was no significant difference in joint score between the DFX and control rats. By day 14 , however, $100 \%$ of the control rats had joint scores of 2 or more compared with only $8 \%$ of rats receiving $100 \mathrm{mg} / \mathrm{kg} \mathrm{DFX}$ and $25 \%$ of rats receiving $200 \mathrm{mg} / \mathrm{kg}$ DFX. Analysis of the foot score data showed a significant reduction in the degree of inflammation from day 14 onwards in the DFX treated animals (Fig. 1). There was no significant difference in joint inflammation between the two doses of DFX nor was there any difference associated with the time of treatment. All control rats were killed by day 22 because of the severity of joint inflammation, but $79 \%$ of rats receiving DFX treatment had mild or no joint inflammation by day 34 .

\section{Histology}

$\mathrm{H} / \mathrm{E}$ stained sections of a random selection of control rat ankle joints showed a florid erosive arthritis. The synovium showed proliferation with a dense infiltration of polymorphs and some histiocytes but an almost complete absence of lymphocytes and plasma cells. Considerable soft tissue oedema was noted. Inflammatory granulation tissue extended into and eroded the subchondral bone, particularly of the tibia, and there was considerable osteoblast and osteoclast activity with bone resorption and reactive bone formation. The articular cartilage was remarkably well preserved. No evidence of vasculitis was seen.

$\mathrm{H} / \mathrm{E}$ sections of DFX treated rat ankle joints chosen at random showed little or no inflammatory changes in most of the tissue studied. On some sections a mild synovitis with soft tissue swelling was observed, but there was no evidence of bone erosion on these sections. The overall histological appearances support our gross 'clinical' assessments of inflammation, indicating a reduction in both the incidence and severity of the joint inflammation associated with DFX treatment.

\section{Microfocal radiography}

Radiography of the other hind foot from each rat reflected histological findings and gross clinical assessment. Microfocal radiography of control joints showed marked destructive lesions of the peripheral metaphyses, consisting of periostitis, osteoporosis (especially of the tibia head) with new bone formation, and considerable soft tissue swelling.

The ankle joints of DFX treated animals showed no evidence of bone changes.

\section{SYSTEMIC DISEASES}

\section{Lymph nodes}

Inguinal lymph nodes from both DFX and saline treated rats showed a similar response to the adjuvant. There was no significant difference in the diameter of nodes (DFX $100 \mathrm{mg} / \mathrm{kg}$ : 3.9 (SD 2.2) mm, DFX $200 \mathrm{mg} / \mathrm{kg}: 5.4(2 \cdot 3) \mathrm{mm}$, saline: $5 \cdot 2(2.0) \mathrm{mm}$; Student's $t$ test), presence or absence of paracortical hyperplasia, sinus histiocytosis, and dilatation, nor in the proportion of nodes in each group showing secondary follicle formation $(69 \%$ of rats treated with DFX showed secondary follicle formation compared with $85 \%$ of rats treated with saline (Mann-Whitney)). Surprisingly, there was no significant difference in the amount of stainable iron in the lymph nodes of rats receiving either dose of DFX and the saline treated rats, the mean iron score in each group being 2. DFX treated rats, however, had significantly less granulomas in the lymph nodes, $31 \%$ of animals showing granulomas compared with $71 \%$ of saline treated rats (Mann-Whitney, $\mathrm{p}<0 \cdot 05$ ). 

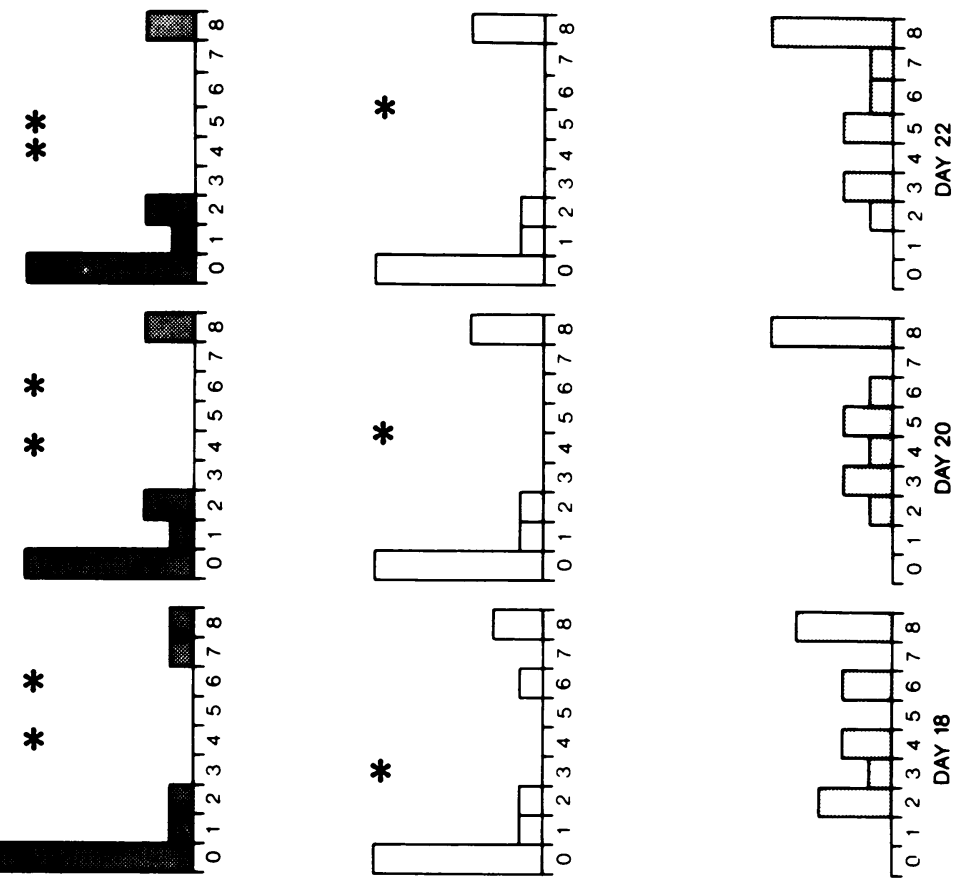

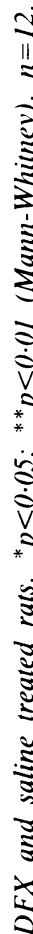
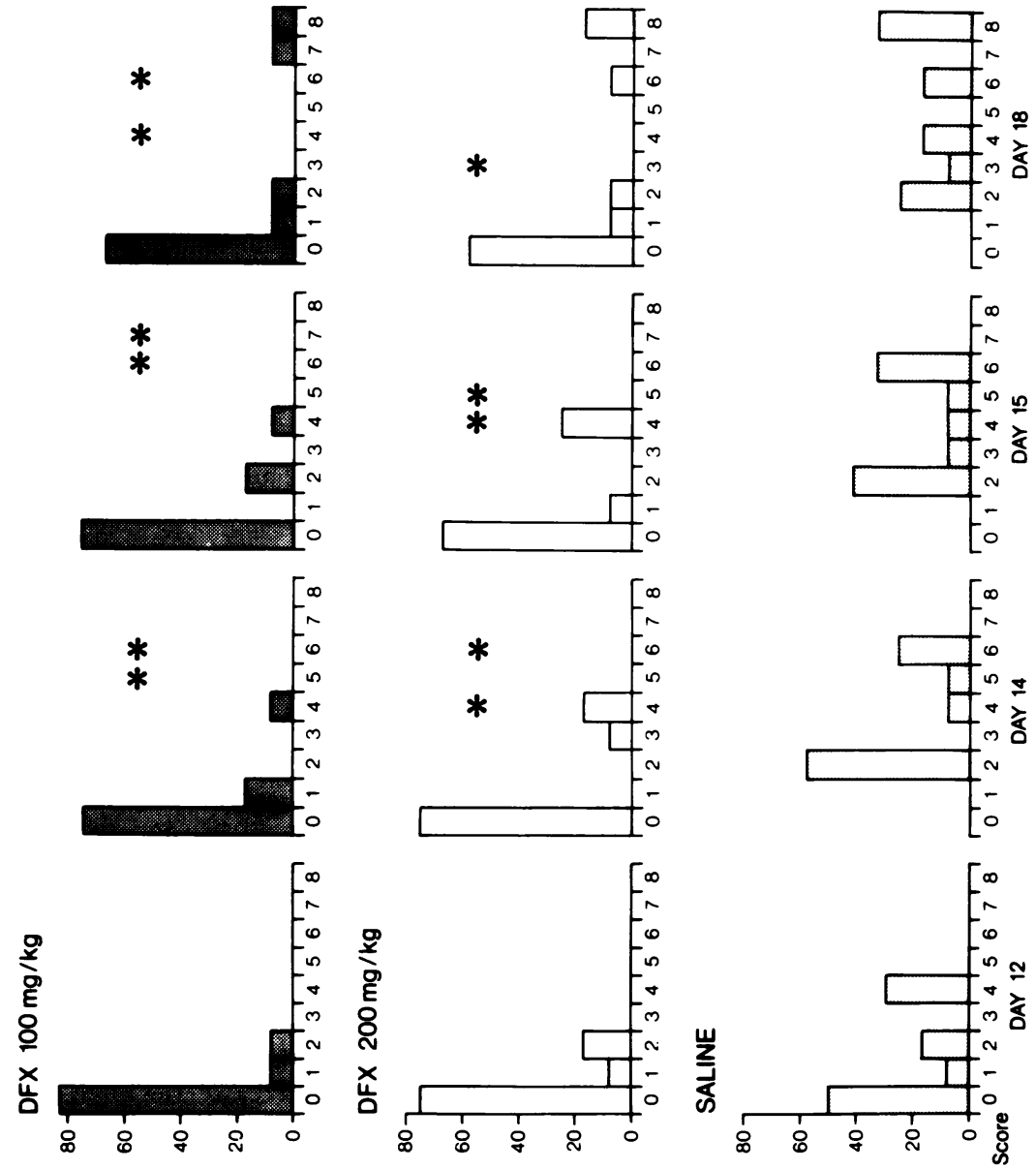

SרYWINY \% 
Liver

Similar pathological changes were noted in both DFX and saline treated animals. There was no significant difference in the number of rats showing granulomas in the liver: $23 \%$ of DFX treated rats compared with $29 \%$ of saline treated rats. Sixty two per cent of DFX treated rats showed foci of inflammatory cells in the liver compared with $71 \%$ of saline treated rats; again these results showed no significant differences (Mann-Whitney). Areas of necrosis in the liver were noted in one DFX treated and one saline treated rat.

\section{$\alpha_{1}$ Acid glycoprotein}

$\alpha_{1}$ Acid glycoprotein levels were found to be raised in DFX treated and saline treated rats as a result of adjuvant disease (DFX $200 \mathrm{mg} / \mathrm{kg}$ : 7.01 (SD $4 \cdot 20 \mathrm{~g} / \mathrm{l}$, DFX $100 \mathrm{mg} / \mathrm{kg}$ : 6.55 (4.90) g/l, saline: 9.89 (4.84) $\mathrm{g} / \mathrm{l}$. Control rats of the same age, strain, and sex with no adjuvant disease had levels of $0.50(0.11) \mathrm{g} / \mathrm{l}$.

There was no significance in the levels of $\alpha_{1}$ acid glycoprotein related to joint inflammation or iron deficiency.

\section{Discussion}

In 1956 Pearson first described a generalised or focal arthritis, synovitis, and tendonitis in rats injected with Freund's complete adjuvant. ${ }^{15}$ Subsequent reports have shown the systemic nature of the disease affecting a range of tissues including joints, eyes, skin, urogenital and gastrointestinal systems. ${ }^{16} 17$ Our results show that the iron chelator DFX when given in a dose capable of reducing reticuloendothelial (hepatic) iron stores will selectively suppress joint inflammation without modifying either the primary inflammatory reaction at the injection site or the systemic sequelae.

Adjuvant disease has been shown to follow a time course characterised by alterations in clinical, pathological, and biochemical parameters. Initially there is an acute local inflammatory response at the site of the injection. ${ }^{18}$ Biochemically this phase is distinguished by an increase in plasma $\alpha_{1}$ acid glycoprotein, reflecting a systemic response to an acute local inflammation. ${ }^{19}$ Iron chelation did not appear to alter this initial response: the size of the tail lesion, the histology, and microfocal radiography of the underlying vertebrae showed similar changes in both DFX treated and control rats; plasma $\alpha_{1}$ acid glycoprotein levels were raised in both groups of animals.

The next phase is characterised by the development of the arthritis which occurs from around day 12 and comprises extensive inflammation of the hind paws followed by the fore paws and spine. ${ }^{20}$ Iron chelation significantly suppressed this aspect of the disease. Rats treated with DFX had a reduced incidence and severity of joint inflammation as assessed by subjective scoring, histology, and microfocal radiography.

Joint inflammation is accompanied by the development of systemic pathology. Granulomas have been observed in the lungs, lymph nodes, spleen, liver, and heart of rats with adjuvant disease, ${ }^{21}$ and hyperplasia of the lymph nodes draining the inoculation site has been reported. ${ }^{13}$ It is thought that the earliest lesions are quite similar histologically in all the affected tissues, including the joints, ${ }^{22}{ }^{23}$ and that rats failing to develop arthritis also fail to develop other lesions. ${ }^{16}$ This is clearly incorrect. Iron chelation did not appear to affect the development of granulomas in the liver or the presence of paracortical hyperplasia, dilatation, and histiocytosis of the sinuses or secondary follicle formation in the draining lymph nodes. These results would suggest that different mechanisms influence joint inflammation as opposed to the systemic lesions.

Like rheumatoid disease the aetiology of adjuvant disease is not firmly established but is thought to involve immune mechanisms either in the development of the disease ${ }^{24}$ or as an exacerbatory influence. ${ }^{25}$ Iron deficiency may impair both humoral $^{26}$ and cell mediated immunity ${ }^{27}$ in rats, and it is therefore possible that iron deficient immune suppression is responsible for the reduction in joint inflammation observed in the DFX treated group. This does not easily explain, however, the presence of systemic disease and especially the changes observed in the lymph nodes of the DFX treated rats.

Free metal ions including iron will catalyse the formation of the hydroxyl radical from the superoxide radical and hydrogen peroxide. ${ }^{28}$ Both reactive oxygen metabolites are produced from stimulated neutrophils, monocytes, and macrophages. ${ }^{29}$ The hydroxyl radical is highly reactive and will damage a variety of biomolecules, including polyunsaturated fatty acids, by the process of lipid peroxidation. Not only is there a tendency for the inflamed joint to accumulate iron as a consequence of microbleeding ${ }^{30}$ but it also provides a suitable environment for iron mediated damage. In rheumatoid arthritis, low synovial fluid $\mathrm{pH}$ is thought to allow the release of iron from transferrin, providing a form of iron capable of catalysing the formation of the hydroxyl radical. ${ }^{31}$ In adjuvant disease we found large numbers of neutrophils and macrophages in the inflamed synovial tissue. This together with the observation that high levels of lipid peroxidation products occur in the synovial fluid of rats with adjuvant disease ${ }^{32}$ suggests a role 
for free radical mediated damage in this model. Iron chelation may specifically protect the joint in adjuvant disease by reducing levels of iron available for the promotion of hydroxyl radical formation and subsequent tissue damage.

High levels of prostaglandin $\mathrm{E}_{2}\left(\mathrm{PGE}_{2}\right)$ have been reported in inflamed tissues ${ }^{33}$ and perfusates ${ }^{34}$ of adjuvant arthritic ankle joints, and the levels measured reflected the severity of swelling. Both periosteal proliferation and bone resorption may be induced by $\mathrm{PGE}_{2},{ }^{35}$ and in vitro studies have shown that iron nitrilotriacetate will augment the release of $\mathrm{PGE}_{2}$ from synovial cells in culture. ${ }^{36}$ Iron chelation may therefore reduce $\mathrm{PGE}_{2}$ production, inhibiting both the periosteal reaction of adjuvant disease and the erosive consequences of synovial inflammation.

In conclusion, iron chelation appears to have a selective effect in reducing joint inflammation in adjuvant disease. We believe this to be a novel finding, which, taken in conjunction with the clinical observation that the iron dextran selectively exacerbates joint inflammation in rheumatoid disease, ${ }^{3}$ suggests a fundamental role for iron in inflammatory joint disease.

This work was supported by grants from West Midlands Regional Health Authority (DRB), British Technology Group (DRB), and the Arthritis and Rheumatism Council for research (CJM, DRB). We are grateful for the assistance of Mr Mike Haddaway and Dr Iain Watt with the microfocal radiography, Mr Mike Robinson and Dr Mike Billingham with the $\alpha_{1}$ acid glycoprotein assay, and Miss J Dowson for secretarial assistance.

\section{References}

1 Muirden K D, Senator G B. Iron in the synovial membrane in rheumatoid arthritis and other joint diseases. Ann Rheum Dis 1968; 27: 38-47.

2 Blake D R, Gallagher P J, Potter A R, Bell M J, Bacon P A. The effect of synovial iron on the progression of rheumatoid disease. Arthritis Rheum 1984; 27: 495-501.

3 Blake D R, Lunec J, Ahern M, Ring E F J, Bradfield J, Gutteridge J M C. Effect of intravenous iron dextran on rheumatoid synovitis. Ann Rheum Dis 1985; 44: 183-8.

4 Sedgwick A D, Blake D R, Winwood P, Moore A R, Al-Duay A Y, Willoughby D A. Studies into the effects of the iron chelator desferrioxamine on the inflammatory process. Eur $J$ Rheumatol Inflamm (in press).

5 Blake D R, Hall N D, Bacon P A, Dieppe P A, Halliwell B, Gutteridge J M C. Effect of a specific iron chelating agent on animal models of inflammation. Ann Rheum Dis 1983; 42: 89-93.

6 Muirden K D, Peace G. Light and electron microscope studies in carrageenan, adjuvant and tuberculin induced arthritis. Ann Rheum Dis 1969; 28: 392-401.

7 Pearson C M. Experimental joint disease: observations on adjuvant induced arthritis. $J$ Chron Dis 1963; 16: 863-74.

8 Kaibara N, Hotokebuchi B, Takagishi K, et al. Pathogenic difference between collagen arthritis and adjuvant arthritis. $J$ Exp Med 1984; 159: 1388-96.

9 Drury R A B, Wallington E A. Carleton's histological technique. 4th ed. Oxford: Oxford University Press, 1976.
10 Currey H L F, Ziff M. Suppression of adjuvant disease in the rat by heterologous antilymphocyte globulin. J Exp Med 1968; 127: 185-203.

11 Newbould B B. Role of lymph nodes in adjuvant induced arthritis in rats. Ann Rheum Dis 1964; 23: 392-6.

12 Ryzewska A G. Influence of lymphatic tissue on the develop ment of adjuvant induced polyarthritis in rats. Ann Rheum 1967; 26: 506-16.

13 Glenn E M, Gray J. Adjuvant induced polyarthritis in ra®: biologic and histologic background. Am J Vet Res 1965; 28 1180-94.

14 Billingham M E J, Gordon A H. The role of the acute phase reaction in inflammation. Agents Actions 1976; 6: 195-200.

15 Pearson C M. Development of arthritis, periarthritis and periostitis in rats given adjuvants. Proc Soc Exp Biol Med 195 91: $95-101$

16 Pearson C M, Waksman B H, Sharp J T. Studies of arthritis añt other lesions induced in rats by injection of mycobacter adjuvant. V. Changes affecting the skin and mucous menbranes. J Exp Med 1961; 113: 485-509.

17 Waksman B H, Billington S J. Studies of arthritis and othe lesions induced in rats by injection of mycobacteria adjuvant Hil lesions of the eye. Arch Ophthalmol 1960; 64: 751-62.

18 Pearson C M, Wood F D. Studies of polyarthritis and othet lesions induced in rats by injection of mycobacterial adjuvanip General, clinical and pathologic characteristics and some modifying factors. Arthritis Rheum 1959; 2: 440-59.

19 Gralla E J, Wiseman E H. The adjuvant arthritic r $\overrightarrow{a 0}$ inflammatory parameters during development and regression gross lesions. Proc Soc Exp Biol Med 1968; 128: 493-5.

20 Pearson C M, Wood F D. Studies of arthritis and other lesiogs induced in rats by injection of mycobacterial adjuvant. VE Pathologic details of the arthritis and spondylitis. Am J Path 1963; 42: 73-95.

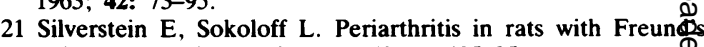
adjuvants. Arthritis Rheum 1960; 3: 485-95.

22 Jones R S, Ward J R. Studies on adjuvant induced polyarthritis in rats. III. Histogenesis of joint and visceral lesions. Arthrgs Rheum 1963; 6: 23-35.

23 Burstein N A, Waksman B H. The pathogenesis of adjuva需 disease in the rat. Histologic study of early lesions. Yale $J$ Biẹl Med 1964; 37: 177-94.

24 Waksman B H, Pearson C M, Sharp J T. Studies of arthritis a and other lesions induced in rats by injection of mycobacteriag adjuvant. II. Evidence that the disease is a disseminated immunologic response to exogenous antigen. J Immunol 1960; 85: 403-17.

25 Billingham M E J. Models of arthritis and the search for antiarthritic drugs. Pharmacol Ther 1983; 21: 389-428.

26 Kochanowski B A, Sherman A R. Decreased antibody formation in iron deficient rat pups. Effect of iron repletion. $A m D>$ Clin Nutr 1985; 41: 278-84.

27 Kochanowski B A, Sherman A R. Cellular growth in ir deficient rats: effect of pre- and post-weaning iron repletiøn. J Nutr 1985; 115: 279-87.

28 Halliwell B, Gutteridge J M C. Oxygen toxicity, oxygen radicals, transition metals and disease. Biochem $J$ 1984; 2 Es: 1-14.

29 Halliwell B, Gutteridge J M C, Blake D R. Metal ions and oxygen radical reactions in human inflammatory joint diseaf?. Philos Trans $R$ Soc Lond [Biol] 1985; 311: 659-71.

30 Morris C J, Blake D R, Wainwright A C, Steven M M. The relationship between iron deposits and tissue damage in the synovium: an ultrastructural study. Ann Rheum Dis 1986; 5 : 21-6.

31 Rowley D, Gutteridge J M C, Blake D R, Farr M, Halliwell Lipid peroxidation in rheumatoid arthritis: thiobarbituric a需 reactive material and catalytic iron salts in synovial fluid from rheumatoid arthritis. Clin Sci 1984; 66: 691-5.

32 Yoshikawa T. Tanaka H, Kanao M. Lipid peroxidation in gat 
adjuvant arthritis and its inhibition by indomethacin. $J$ Appl Biochem 1983; 5: 382-7.

33 Barbier A, Navarro J, Breliere J C, Roncucci R. Biochemical and clinical changes in rats with developing adjuvant arthritis. Agents Actions 1984; 15: 103-10.

34 Parnham M J, Bonta I L, Adolfs M J. Cyclic AMP and prostaglandin $\mathrm{E}$ in perfusates of rat hind paws during the development of adjuvant arthritis. Ann Rheum Dis 1978; 37: 218-24.
35 Galasko C S B, Bennett A. Relationships of bone destruction in skeletal metastases to osteoclast activation and prostaglandins. Nature 1976; 263: 508-10.

36 Okasaki I, Brinckerhoff C E, Sinclair J F, Sinclair P R, Bronkowsky H L, Harris E D. Iron increases collagenase production by rabbit synovial fibroblasts. J Lab Clin Med 1981; 97: $396-402$. 